\title{
Silencing of long noncoding RNA H19 alleviates pulmonary injury, inflammation and fibrosis in acute respiratory distress syndrome rats through regulating MicroRNA-423-5p
}

\section{Xianyu Mu}

Yantai Yuhuangding Hospital

Hongrong Wang

Yantai Yuhuangding Hospital

Haiyong Li ( $\square$ lhyqch@163.com )

Yantai Yuhuangding Hospital https://orcid.org/0000-0001-8740-2229

\section{Research article}

Keywords: H19, LPS-induced ARDS, pulmonary inflammation, pulmonary fibrosis, miR-423-5p

Posted Date: January 23rd, 2020

DOl: https://doi.org/10.21203/rs.2.21767/v1

License: (c) (i) This work is licensed under a Creative Commons Attribution 4.0 International License.

Read Full License 


\section{Abstract}

Background: This study aimed to explore the function of long noncoding RNA H19 (H19) on pulmonary injury, inflammation and fibrosis in lipoproteins (LPS)-induced acute respiratory distress syndrome (ARDS) rats.

Methods: The LPS-induced ARDS rat model was established by intratracheal instillation with $2 \mathrm{mg} / \mathrm{kg}$ LPS. QRT-PCR was performed to detect the expression of H19, miR-423-5p, tumor necrosis factor- $a$ (TNFa), interleukin (IL)-1 $\beta$, IL-6, monocyte chemoattractant protein (MCP)-1 and vascular endothelial growth factor (VEGF). Histology score was detected by hematoxylin-eosin (HE) staining. Enzyme-linked immunosorbent assay (ELISA) was used to detect the levels of proinflammatory cytokines and the concentration of VEGF in bronchoalveolar lavage fluid (BALF). The protein expression of fiber factors was measured by western blot. The degree of fibrosis was observed by masson-trichrome staining. Dualluciferase reporter assay was used to determine the binding site between miR-423-5p and H19.

Results: The expression of H19 was significantly increased, while miR-423-5p was decreased in LPSinduced ARDS rats. Silencing of H19 decreased the mRNA expression of TNF- $\alpha$, IL-1 $1 \beta$, IL-6, MCP-1 and VEGF in LPS-induced ARDS rats, and decreased the levels of TNF-a, IL-1 $\beta$, IL-6and the concentration of VEGF in BALF, histology score of LPS-induced ARDS rats. H19 inhibition also decreased the fibrosis score and the proteins expression of fiber factors of LPS-induced ARDS rats. Furthermore, miR-423-5p eliminated the effect of $\mathrm{H} 19$ on LPS-induced MH-S cells.

Conclusions: Silencing of $\mathrm{H} 19$ ameliorated the pulmonary injury, inflammation and fibrosis of LPSinduced ARDS through regulating miR-423-5p, which may be a promising therapeutic strategy to treat ARDS.

\section{Background}

Acute respiratory distress syndrome (ARDS) is characterized by severe hypoxaemia, pulmonary inflammation, alveolar edema and pulmonary fibrosis [1]. ARDS has a mortality of $20 \%$ $50 \%$ with poor prognosis worldwide [2]. ARDS patients require mechanical ventilatory support that carries a high financial cost [3]. There are extremely few effective treatments for ARDS mainly due to its complex pathogenesis and timely diagnosis except lung preservation strategies [4, 5]. Herein, it is necessary to explore new strategies or promising biomarkers for ARDS treatment and diagnosis.

Long noncoding RNAs (IncRNAs) are a class of noncoding RNA transcripts with a minimum length of 200 nucleotides and limited protein-coding capacity [6, 7]. Increasing researches demonstrate that IncRNAs could take part in the pathogenesis of many pulmonary diseases, such as IncRNA MALAT1 in acute lung injury (ALI) [8], IncRNAPCAT29 in pulmonary fibrosis [9] and IncRNA TUG1 in chronic obstructive pulmonary disease [10]. LncRNA H19 (H19) as one of the IncRNAs has been found to be involved in several pulmonary diseases. For instance, knockdown of $\mathrm{H} 19$ alleviates pulmonary fibrosis through regulating the miR-196a/COL1A1 axis [11]. Zhang et al. suggested that H19 can promote epithelial 
mesenchymal transition (EMT) in lung cancer through targeting miR-484 [12]. However, the underlying mechanism and biological influence of $\mathrm{H} 19$ in the regulation of ARDS progression remain still limited.

LncRNAs modulate the expression of mRNAs via regulating its translation and degradation through interacting with microRNAs (miRNAs) [13]. MiRNAs are series of small, noncoding RNAs with a length of $1825 \mathrm{nt}$ [14]. Numerous studies have proved the key function of miRNAs in the progresses of ALI. MiR27a alleviates the inflammatory responses of pulmonary in LPS-induced ALI via regulating TLR4/MyD88/NF-KB pathway [15]. Overexpression of miR-125b ameliorates the inflammation and histopathology changes of pulmonary in LPS-induced ALI [16]. MiR-144-3p protects mice from LPSinduced ALI via reducing lung epithelial cell apoptosis [17]. ARDS is a severe clinical syndrome of ALI, and affects both surgical and medical patients [18]. However, the mediatory relationship between $\mathrm{H} 19$ and miR-423-5p on ARDS remains unclear.

Here, we determined the function of $\mathrm{H} 19$ on the pulmonary injury, inflammation and fibrosis in lipoproteins (LPS)-induced ARDS rats. We found that miR-423-5p is a target gene of H19. Then we explored the regulatory effect of miR-423-5p on the expression of proinflammatory cytokines, CAMs, chemotactic factors, VEGF and the proteins expression of fiber factors in LPS-induced MH-S cells. Our research may discover a hopeful therapeutic target for ARDS, and broaden our horizons of the underlying mechanisms responsible for ARDS treatment.

\section{Methods}

\section{Animals}

Twenty male wistar rats $(180 \pm 20 \mathrm{~g})$ were purchased from Institute of Zoology, Chinese Academy of Sciences (Beijing, China). Rats were fed standard chow and water, and maintained under the light/dark cycle (12 h/12 h). This study was performed with the approval of our hospital Animal Ethics Committee.

\section{LPS-induced ARDS model in rats}

LPS-induced ARDS rat model was established by intratracheal instillation with $2 \mathrm{mg} / \mathrm{kg}$ LPS (SigmaAldrich, St. Louis, MO, USA) and were divided into ARDS, ARDS + sh-negative control (NC) and ARDS + sh$\mathrm{H} 19$ group (5 rats in each group). ARDS rats were then intravenously injected with $2 \times 10^{7} \mathrm{TU} / \mathrm{ml}$ sh-H19 (ARDS + sh-H19 group) or $2 \times 10^{7} \mathrm{TU} / \mathrm{ml}$ sh-NC (ARDS + sh-NC group) at $48 \mathrm{~h}$ post LPS induction. After

$24 \mathrm{~h}$ of treatment, rats were anesthetized with $50 \mathrm{mg} / \mathrm{kg}$ pentobarbital sodium and sacrificed by cervical dislacation. Sham group rats $(n=5)$ were intratracheally instilled with an equal volume of normal saline (NS). Blood samples, lung tissues and bronchoalveolar lavage fluid (BALF) were collected for the future experiments.

\section{Histopathology detection by hematoxylin-eosin (HE) staining}


Lung samples were fixed in $4 \%$ paraformaldehyde for $24 \mathrm{~h}$, embedded in paraffin, cut into sections at $6 \mu \mathrm{m}$ thickness, and stained with HE staining. By means of light microscopy, the histopathological change of lung tissues was observed. The histology score of lung tissues was evaluated as previously described [19].

\section{Analysis of arterial blood gas and ratio of lung wet/dry (W/D) weight}

Samples of arterial blood were obtained from carotid artery. Partial pressure of arterial oxygen $\left(\mathrm{PaO}_{2}\right)$ and partial pressure of arterial carbon dioxide $\left(\mathrm{PaCO}_{2}\right)$ were detected by automatic blood gas analyzer (Radiometer, Copenhagen, Denmark). Lungs were weighed and subsequently dried in a $60{ }^{\circ} \mathrm{C}$ oven for 72 hours. The ratio of lung W/D weight represented tissue edema.

\section{Assay of BALF}

BALF collection was performed through lavaging the lungs and airways three times with NS. The levels of total protein (TP), tumor necrosis factor- $\alpha$ (TNF-a), interleukin (IL)-1 $\beta$ and IL- 6 in BALF were examined using enzyme-linked immunosorbent assay (ELISA) kits (R\&D systems, Inc., Mineapolis, USA).

\section{Cell culture}

MH-S and MLE-12 cells were obtained from American Type Culture Collection (ATCC). All cell lines were cultured in Roswell Park Memorial Institute (RPMI)-1640 (GIBCO, Erie, NY, USA) with 10\% fetal bovine serum (FBS, Invitrogen, Carlsbad, NY, USA) at $37^{\circ} \mathrm{C}$ containing $5 \% \mathrm{CO}_{2}$.

\section{Cell transfection}

The si-H19, si-NC, miR-423-5p inhibitor and inhibitor negative control (INC) were obtained from Shanghai Genepharma (Shanghai, China). MH-S and MLE-12 cells were stimulated with $100 \mathrm{ng} / \mathrm{ml}$ LPS for $24 \mathrm{~h}$. Then, MH-S and MLE-12 cells were transfected with above agents using Lipofectamine 3000 reagent (Invitrogen).

\section{Western blot}

Lung tissues were lysed by ice-cold lysis buffer to obtain total protein. The concentrations of total protein were detected by bicinchoninic acid (BCA) protein concentration assay kit (Pierce, Rockford, IL, USA). Protein samples were separated in sodium dodecyl sulfate polyacrylamide gel electrophoresis (SDSPAGE), and transferred onto polyvinylidene fluoride (PVDF) membrane. Then membranes were incubated with primary antibody overnight at $4{ }^{\circ} \mathrm{C}$. The antibodies used in this study were as follows: anti- $\beta$-actin (1:1000, ab8227, Abcam, Cambridge, MA, USA), anti-vimentin (1:1000, ab92547, Abcam), anti-a-smooth muscle actin (a-SMA) (1:1000, ab124964, Abcam) and anti-E-cadherin (1:1000, ab76319, Abcam). The membranes were then incubated with horseradish peroxidase (HRP)-labeled goat anti-rabbit lgG (1:5000, ab6712, Abcam) secondary antibody for $1 \mathrm{~h}$ at room temperature. Finally, the protein bands were visualized by enhanced chemiluminescence (ECL) exposure solution, and quantified by a gel imaging system (UVP, Upland, CA, USA). $\beta$-actin was introduced as the internal reference. 


\section{Quantitative real time polymerase chain reaction (qRT-PCR)}

The expression of H19, miR-423-5p, TNF-a, IL-1 $\beta$, IL-6, intercellular adhesion molecule (ICAM)-1, vascular cell adhesion molecule (VCAM)-1, monocyte chemoattractant protein (MCP)-1 and vascular endothelial growth factor (VEGF) was detected by qRT-PCR. The total RNA of cells and tissues was extracted using TRIZOL reagent (Invitrogen) and was reverse-transcribed into cDNA by Takara PrimeScript RT reagent kit (Takara, Otsu, Japan). PCR reaction was performed on ABI 7500HT Fast Real-Time PCR System (Applied Biosystems, Waltham, MA, USA) with the following conditions: $95^{\circ} \mathrm{C}$ for $3 \mathrm{~min}, 40$ cycles of $95^{\circ} \mathrm{C}$ for $15 \mathrm{~s}$ and $60^{\circ} \mathrm{C}$ for $30 \mathrm{~s}$. Relative expression was calculated by the $2^{-\Delta \Delta \mathrm{Ct}}$ method. $\mathrm{U} 6$ and $\beta$-actin were used for the normalization. The primer sequences were shown in Table 1. 
Table 1

Primer sequences used in quantitative real-time PCR

\begin{tabular}{|c|c|}
\hline Name of primer & Sequences $\left(5^{\prime}-3^{\prime}\right)$ \\
\hline VEGF-F & GTAACGATGAAGCCCTGGAGTG \\
\hline VEGF-R & CGTCTGCGGATCTTGGACAAAC \\
\hline$\beta$ actin- $F$ & TGCTGTCCCTGTATGCCTCTG \\
\hline$\beta$ actin- $R$ & CTTTGATGTCACGCACGATTT \\
\hline IL-6-F & GGAAATCGTGGAAATGAG \\
\hline IL-6-R & AGGACTCTGGCTTTGTCT \\
\hline IL-1 $\beta-F$ & GCCCTAAACAGATGAAGTGCTC \\
\hline IL-1 $1 \beta-R$ & GAACCAGCATCTTCCTCAG \\
\hline ICAM)-1-F & CAAACGGGAGATGAATGG \\
\hline ICAM-1-R & TGGCGGTAATAGGTGTAAAT \\
\hline VCAM-1-F & CGGTCATGGTCAAGTGTTTG \\
\hline VCAM-1-R & GAGATCCAGGGGAGATGTCA \\
\hline MCP-1-F & ATGCAGGTCTCTGTCACGCT \\
\hline MCP-1-R & GGTGCTGAAGTCCTTAGG \\
\hline $\mathrm{H} 19-\mathrm{F}$ & GAATTCAGTTAGAAAAAGCCCGGGCT \\
\hline H19-R & GCGGCCGCTTTGCTGTAACAGTGTTTATTG \\
\hline miR-423-5p-F & GGGAGCAAGATGGCGATTC \\
\hline miR-423-5p-R & CССTCAAACTTCGGGCTTC \\
\hline U6-F & TGCGGGTGCTCGCTTCGGCAGC \\
\hline U6-R & CCAGTGCAGGGTCCGAGGT \\
\hline
\end{tabular}

\section{Immunohistochemistry}

Sections of lung tissue were dewaxed in xylene, dehydrated in graded alcohol, and incubated in $3 \% \mathrm{H}_{2} \mathrm{O}_{2}$ for $0.5 \mathrm{~h}$. The sections were blocked with normal goat serum, followed by anti-vascular endothelial growth factor (VEGF) (1:100; ab185238, Abcam) antibody incubation. Then the sections were incubated 
with HRP-labeled goat-anti rabbit IgG (1:1000, ab6721, Abcam) secondary antibody. Immunohistochemistry was captured by a digital microscope (Nikon Eclipse 80i, Nikon, Tokyo, Japan).

\section{Masson-trichrome staining}

The 6- $\mu \mathrm{m}$ thickness paraffin-embedded tissues sections of lung were stained with masson-trichrome. The ratio of fibrotic area was observed using light microscopy and the fibrosis score was graded as previously described: 0 , absent, appears normal (-); 1 , light (+); 2 , moderate $(++) ; 3$, strong (+++); 4, intense (++++) [20].

\section{Dual-luciferase reporter assay}

The potential binding sites of miR-423-5p and H19 were predicted according to StarBase3.0. The H19-Wt and $\mathrm{H} 19-$ Mut were cloned and combined with psiCHECK-2 vectors (Promega, Madison, WI, USA). H19-Wt or H19-Mut was co-transfected with mimics-NC or miR-423-5p mimics (Shanghai Genepharma) into MLE12 cells with Lipofectamine 3000 (Invitrogen). The luciferase activity was detected by Dual-luciferase reporter gene assay system (Promega).

\section{Statistical analysis}

Statistical analysis was performed with SPSS 23.0 (SPSS Inc., Chicago, IL, USA) and GraphPad Prism software 7.0 (San Diego, CA, USA). Data was presented as mean \pm SD. The differences between various groups were analyzed by one-way ANOVA followed by the multiple comparisons test. The data of two groups were assessed using Student's t-test. A P value $<0.05$ was considered statistically significant.

\section{Results}

\section{Silencing of $\mathrm{H} 19$ alleviates pulmonary injury of LPS-induced ARDS}

LPS-induced ARDS model in rat was established by induction of LPS. QRT-PCR showed that the expression of H19 was markedly up-regulated in the ARDS group $(P<0.01)$ (Fig. 1A). As shown in Fig. 1B, the expression of $\mathrm{H} 19$ was markedly down-regulated in the ARDS + sh-H19 group compared with the ARDS group $(\mathrm{P}<0.001)$, suggesting that the transfection was succeed, and the transfection of sh-H19 markedly down-regulated the relative expression of $\mathrm{H} 19$. The histopathological change and histology score of lung tissues were observed by HE staining. Compared with the sham group, the inflammatory cells infiltration and interstitial edema were obviously in the ARDS group. Treatment with sh-H19 markedly alleviated the inflammatory cells infiltration and interstitial edema (Fig. 1C). By contrast to the sham group, the histology score was markedly elevated in the ARDS group $(P<0.001)$ (Fig. 1D). Silencing of $\mathrm{H} 19$ markedly reduced the histology score of lung tissues $(\mathrm{P}<0.01)$ (Fig. 1D). Additionally, the level of blood $\mathrm{PaO}_{2}$ was markedly reduced and $\mathrm{PaCO}_{2}$, ratio of lung W/D weight and lung edema score were increased in the ARDS group comparing with the sham group $(\mathrm{P}<0.001)$ (Fig. 1E-H). H19 inhibition 
markedly elevated $\mathrm{PaO}_{2}$ level and decreased $\mathrm{PaCO}_{2}$, ratio of lung W/D weight and lung edema score $(\mathrm{P}<$ 0.05 or $\mathrm{P}<0.01)($ Fig. 1E-H).

\section{H19 inhibition attenuates pulmonary inflammation of LPS- induced ARDS rats}

To evaluate the effect of $\mathrm{H} 19$ inhibition on pulmonary inflammation of LPS-induced ARDS rats, we examined the content of TP, TNF-a, IL- 6 and IL-1 $1 \beta$ by ELISA. Compared with the sham group, the levels of TP, TNF- $a, I L-6$ and IL-1 $\beta$ in BALF were significantly increased in the ARDS group $(P<0.001)$. The above indexes in BALF in the ARDS + sh-H19 group were markedly lower than that in the ARDS group $(P<0.001)$ (Fig. 2A-D). The mRNA expression levels of TNF-a, IL-6, IL-1 3 , ICAM-1, VCAM-1 and MCP-1 in lung tissues was measured by qRT-PCR. The mRNA expression levels of TNF-a, IL-6, IL-1 $\beta$, ICAM-1, VCAM- 1 and MCP-1 in lung tissues was significantly up-regulated in the ARDS group by contrast to the sham group. Silencing of $\mathrm{H} 19$ markedly down-regulated the above indexes in lung tissues $(\mathrm{P}<0.001)$ (Fig. 2E-J).

Immunohistochemical assay showed that staining intensity of VEGF was markedly increased in the ARDS group compared with the sham group. The staining intensity of VEGF in the ARDS + sh-H19 group was markedly lower than that in the ARDS group $(P<0.001)$ (Fig. $2 \mathrm{~K}$ and $\mathrm{L})$. The concentration of VEGF in BALF and the mRNA expression level of VEGF in lung tissues were detected by ELISA and qRT-PCR, respectively. The concentration of VEGF in BALF and the mRNA expression level of VEGF in lung tissues was markedly up-regulated in the ARDS group compared with the sham group $(P<0.001)$. Knockdown of $\mathrm{H} 19$ markedly reduced the concentration of VEGF in BALF and mRNA expression level of VEGF in lung tissues $(P<0.01)$ (Fig. $2 \mathrm{M}$ and $N)$.

\section{Knockdown of H19 ameliorates pulmonary fibrosis of LPS- induced ARDS rats}

To evaluate the effect of $\mathrm{H} 19$ knockdown on pulmonary fibrosis of LPS-induced ARDS rats, we examined the E-cadherin, vimentin and a-SMA proteins expression levels using western blot. Compared with the sham group, the protein expression level of E-cadherin in lung tissues were markedly inhibited in the ARDS group. Knockdown of $\mathrm{H} 19$ markedly elevated the protein expression level of E-cadherin in lung tissues $(P<0.001)$ (Fig. 3A-C). The proteins expression levels of vimentin and a-SMA was contrary with Ecadherin $(P<0.001)$ (Fig. 3D). Masson-trichrome staining showed that the ratio of fibrotic area and fibrosis score in lung tissues were significantly elevated in the ARDS group compared with the sham group and the sh-H19 injection markedly inhibited the promoting impacts of ARDS on the ratio of fibrotic area and fibrosis score $(P<0.001)$ (Fig. 3E and F).

\section{MiR-423-5p is a direct target of $\mathrm{H} 19$}

StarBase3.0 was utilized to predict the relationship between miR-423-5p and $\mathrm{H} 19$, and the sequence of binding site was showed in Fig. 4A. Dual-luciferase reporter assay suggested the luciferase activity of 
MH-S and MLE-12 cells co-transfected with miR-423-5p mimics and H19 Wt was significantly decreased $(P<0.001)$ while there was none difference after miR-423-5p mimics and H19 Mut co-transfection ( $P>$ 0.05) (Fig. 4B and C). The expression of miR-423-5p in lung tissues, lung cells (MH-S and MLE-12 cells) and $\mathrm{H} 19$ in lung cells were measured by qRT-PCR. The expression of miR-423-5p in lung tissues was significantly down-regulated in the ARDS group. Silencing of H19 markedly promoted the expression of miR-423-5p in lung tissues $(P<0.001)$ (Fig. 4D). Compared with mock group, the expression of $\mathrm{H} 19$ in $\mathrm{MH}-\mathrm{S}$ and MLE-12 cells was significantly down-regulated in the si-H19 group $(\mathrm{P}<0.001)$ (Fig. 4E and F). Knockdown of H19 increased the expression of miR-423-5p in MH-S and MLE-12 cells $(P<0.001)(F i g .4 G$ and $\mathrm{H}$ ).

\section{MiR-423-5p eliminates the effect of H19 on the LPS- stimulated $\mathrm{MH}-\mathrm{S}$ cells}

In order to explore the potential mechanism of H19/miR-423-5p axis, we firstly performed qRT-PCR analysis for $\mathrm{H} 19$ and miR-423-5p levels in MH-S cells. By contrast to the control group, the expression of $\mathrm{H} 19$ in $\mathrm{MH}-\mathrm{S}$ cells was markedly up-regulated in the LPS group $(\mathrm{P}<0.001)$ (Fig. 5A), and miR-423-5p was down-regulated $(\mathrm{P}<0.001$ ) (Fig. 5B). The expression of $\mathrm{H} 19$ in $\mathrm{MH}-\mathrm{S}$ cells in the LPS + si-H19 group was lower than that in the LPS group $(P<0.001)$ (Fig. 5C). The transfection of miR-423-5p inhibitor inhibited the expression of miR-423-5p in $\mathrm{MH}-\mathrm{S}$ cells $(\mathrm{P}<0.001)$ (Fig. 5D). After detection of inflammatory factor and pulmonary fibrosis associated protein, we found that the mRNA expression levels of TNF-a, IL-6, IL$1 \beta$, ICAM-1, VCAM-1, MCP-1 and VEGF and the proteins expression levels of vimentin and a-SMA in MH-S cells was markedly decreased in the INC + si-H19 group than those in the INC + si-NC group $(P<0.01)$ (Fig. 5E-K). The above indexes in $\mathrm{MH}-\mathrm{S}$ cells were markedly increased in the miR-423-5p inhibitor + si-NC group comparing with the INC + si-NC group $(P<0.001)$. The transfection of miR-423-5p inhibitor markedly rescued the inhibiting effect of si-H19 on the above indexes in MH-S cells $(P<0.001)(F i g .5 E-K)$. The protein expression level of E-cadherin in MH-S cells was contrary with vimentin and a-SMA $(\mathrm{P}<$ 0.001) (Fig. 5J).

\section{Discussion}

$\mathrm{H} 19$ is one of the most highly abundant and conserved transcripts in the mammalian development [21]. Prior reports have shown that $\mathrm{H} 19$ is significantly up-regulated in lung cancers, including lung adenocarcinoma [22] and non-small cell lung cancer [23]. However, the relationship between H19 expression and ARDS remains unknown. Here, we displayed that the expression of $\mathrm{H} 19$ was markedly increased in lung tissues of LPS-induced ARDS, indicating that H19 may play a vital role in ARDS.

LncRNAs participate in the inflammatory epithelial injury and hypoxic in pulmonary diseases $[24,25]$. The injury of pulmonary in ARDS is characterized by inflammation, elevated alveolar-capillary permeability and alveolar edema [26]. Hypoxemia is delimited as a ratio of $\mathrm{PaO}_{2} / \mathrm{FiO} 2$ less than $200 \mathrm{mmHg}$ in ARDS [27]. The lung W/D weight ratio is dramatically increased in ARDS [28]. The degree of lung edema is estimated via examining the ratio of lung W/D weight [29]. In this study, silencing of H19 also decreased 
the histopathological change of lung tissue, the histology score, $\mathrm{PaCO}_{2}$, ratio of lung W/D weight and lung edema score, and increased the $\mathrm{PaO}_{2}$ in LPS-induced ARDS. Our finding suggested that silencing of H19 could ameliorate lung injury in LPS-induced ARDS.

The pathogenesis of early steps of ARDS is induced by exaggerated inflammatory response [30]. The concentration of TP in BALF is a surrogate endpoint for severity of lung injury in ARDS [31]. Additionally, the development of ARDS is related to the secretion of proinflammatory cytokines, which are important mediators causing organ dysfunction, increased vascular permeability, endothelial destruction and angiogenesis [32-34]. CAMs (cell adhesion molecules), such as ICAM-1 and VCAM-1 are involved in the complex processes of inflammation of ARDS [35]. MCP-1 is required for the regulation of lung inflammation called chemotactic factors, high level of MCP-1 in ARDS patients is correlated with poor prognosis [36]. In this study, silencing of H19 markedly reduced the level of TP, the levels of proinflammatory cytokines and the expression of ICAM-1, VCAM-1 and MCP-1 in lung tissues of LPSinduced ARDS. Taken together, our finding indicated that silencing of $\mathrm{H} 19$ could alleviate inflammation through inhibiting the expression of proinflammatory cytokines, CAMs and chemotactic factors.

The last phase of the ARDS is characterized by the excessive fibroproliferation of lung connective tissues [37]. EMT is considered as a source of fibroblasts in the progress of pulmonary fibrosis [38]. The expression of vimentin, a-SMA and E-cadherin is the EMT markers [39]. Additionally, knockdown of IncPFAR protects against pulmonary fibrosis by decreasing the fibrotic area and fibrosis-related proteins in idiopathic pulmonary fibrosis (IPF) mice [40]. Silencing of IncRNA MALAT1 ameliorates pulmonary fibrosis through attenuating the process of EMT in HBE and A549 cells [41]. Consistently, our observations revealed that silencing of $\mathrm{H} 19$ markedly elevated the expression of vimentin and a-SMA, reduced the expression of E-cadherin the ratio of fibrotic area and fibrosis score in lung tissues of LPSinduced ARDS, suggesting that silencing of $\mathrm{H} 19$ could alleviate pulmonary fibrosis in LPS-induced ARDS.

Several studies indicate that miRNAs expression is down-regulation in pulmonary diseases, such as miR101 in IPF [42], miR-503 in chronic obstructive pulmonary disease [43] and miR-1246 in ALI [44]. Previous research has proved that miRNAs participate in alleviate pulmonary inflammation and pulmonary fibrosis. Up-regulation of miR-146a attenuates pulmonary inflammation through reducing the expression of proinflammatory cytokines in alveolar macrophage (AM) NR8383 cells [45]. Transfection of alveolar macrophage with miR-132 markedly attenuates inflammatory responses, and inhibits the cell growth induced by LPS [46]. MiR-200b/c alleviates pulmonary fibrosis, decreased the proteins expression of vimentin and a-SMA, and increased E-cadherin in LPS-induced pulmonary fibrosis [20]. Similar to previous studies, after verified the targeting relationship between $\mathrm{H} 19$ and miR-423-5p, we found that the miR-423-5p inhibitor markedly rescued the inhibiting effect of si-H19 on the proinflammatory cytokines, CAMs, chemotactic factors and fiber factors in LPS-induced MH-S. All above dates indicated that miR423-5p was a direct target of $\mathrm{H} 19$ and miR-423-5p might eliminate the effect of H19 on the MH-S cells.

\section{Conclusions}


In summary, our finding displayed that $\mathrm{H} 19$ was increased in lung tissues of LPS-induced ARDS rats and LPS-induced MH-S cells. Silencing of $\mathrm{H} 19$ ameliorated the pulmonary injury, inflammation and fibrosis of LPS-induced ARDS rats through regulating miR-423-5p. H19 may act as a potential therapeutic target for ARDS.

\section{Declarations}

Ethics approval and consent to participate: This study was conducted after obtaining local ethical committee approval and written informed consent from Yantai Yuhuangding Hospital of Shandong Province

Consent for publication: All authors approved to publish this study

Availability of data and materials: The datasets used and analysed during the current study are available from the corresponding author on reasonable request

Competing interests: The authors declare that they have no competing interests

Authors' contributions: XYM conducted data Conceptualization, management and formal data analysis. HRW conducted research methodology validation and project management HYL performed the resources,supervision and validation. All authors Wrote - original draft . All authors read and approved the final manuscript.

Acknowledgements: Not applicable

Funding: No funding was obtained for this study.

\section{Abbreviations}

lipoproteins (LPS)

acute respiratory distress syndrome (ARDS)

monocyte chemoattractant protein (MCP)

vascular endothelial growth factor (VEGF)

hematoxylin-eosin (HE)

Enzyme-linked immunosorbent assay (ELISA)

bronchoalveolar lavage fluid (BALF)

Long noncoding RNAs (IncRNAs) 
acute lung injury (ALI)

epithelial mesenchymal transition (EMT)

microRNAs (miRNAs)

negative control (NC)

normal saline (NS)

bronchoalveolar lavage fluid (BALF)

total protein (TP)

inhibitor negative control (INC)

bicinchoninic acid (BCA)

sodium dodecyl sulfate polyacrylamide gel electrophoresis (SDS-PAGE)

polyvinylidene fluoride (PVDF)

horseradish peroxidase (HRP)

enhanced chemiluminescence (ECL)

intercellular adhesion molecule (ICAM)

vascular cell adhesion molecule (VCAM)

\section{References}

1. Chua F, Bellingan GJ: Acute respiratory distress syndrome: fibrosis fast and furious. Clinical Intensive Care 2011.

2. Villar J, Blanco J, Kacmarek RM: Current incidence and outcome of the acute respiratory distress syndrome. Current Opinion in Critical Care 2015, 22(1):1.

3. Petrucci $N$, Feo CD: Lung protective ventilation strategy for the acute respiratory distress syndrome. Cochrane Database of Systematic Reviews 2013, 2(2):CD003844.

4. Terminella L, Sharma G: Diagnostic Studies in Patients With Acute Respiratory Distress Syndrome. 2007, 18(1):2-7.

5. Fanelli V, Vlachou A, Ghannadian S, Simonetti U, Zhang H: Acute respiratory distress syndrome: New definition, current and future therapeutic options. Journal of Thoracic Disease 2013, 5(3):326-334.

6. Lorenzen JM: Long noncoding RNAs. Der Nephrologe 2016. 
7. Guttman M, Amit I, Garber M, French C, Lin MF, Feldser D, Huarte M, Zuk O, Carey BW, Cassady JP: Chromatin signature reveals over a thousand highly conserved large non-coding RNAs in mammals. Nature, 458(7235):223-227.

8. Dai L, Zhang G, Zhe C, Xi W, Jia L, Jing X, Wang H, Rui Z, Meng L, Jiang T: Knockdown of LncRNA MALAT1 contributes to the suppression of inflammatory responses by up-regulating miR-146a in LPS-induced acute lung injury. Connective Tissue Research 2018(2):1-12.

9. Liu X, Gao S, XU H: IncRNAPCAT29 inhibits pulmonary fibrosis via the TGF- $\beta 1$-regulated RASAL1/ERK1/2 signal pathway. Molecular Medicine Reports 2018, 17(6).

10. Tang W, Zhenyu S, Jiang G, Shenghua S: Screening of long non-coding RNA and TUG1 inhibits proliferation with TGF- $\beta$ induction in patients with COPD. International Journal of Chronic Obstructive Pulmonary Disease, Volume 11:2951-2964.

11. Lu Q, Guo Z, Xie W, Jin W, Zhu D, Chen S, Ren T: The IncRNA H19 Mediates Pulmonary Fibrosis by Regulating the miR-196a/COL1A1 Axis. Inflammation.

12. Zhang Q, Li X, Li X, Li X, Chen Z: LnCRNA H19 promotes epithelial-mesenchymal transition (EMT) by targeting miR-484 in human lung cancer cells. Journal of Cellular Biochemistry.

13. Wu T, Qu L, He G, Tian L, Li L, Zhou H, Jin Q, Ren J, Wang Y, Wang J: Regulation of laryngeal squamous cell cancer progression by the IncRNA H19/miR-148a-3p/DNMT1 axis. Oncotarget, 7(10).

14. Bartel DP: MicroRNAs: Target Recognition and Regulatory Functions. 136(2):0-233.

15. Ju M, Liu B, He H, Gu Z, Liu Y, Su Y, Zhu D, Cang J, Luo Z: MicroRNA-27a alleviates LPS-induced acute lung injury in mice via inhibiting in fl ammation and apoptosis through modulating TLR4/MyD88/NFkappaB pathway. Cell Cycle 2018, 17(16):2001-2018.

16. Guo Z, Gu Y, Wang C, Zhang J, Shan S, Gu X, Wang K, Han Y, Ren T: Enforced expression of miR-125b attenuates LPS-induced acute lung injury. Immunology Letters, 162(1):18-26.

17. Li H, Shi H, Gao M, Ma N, Sun R: Long non-coding RNA CASC2 improved acute lung injury by regulating miR-144-3p/AQP1 axis to reduce lung epithelial cell apoptosis. Cell \& Bioscience, 8(1):15.

18. Ware, Lorraine: Pathophysiology of Acute Lung Injury and the Acute Respiratory Distress Syndrome. Seminars in Respiratory and Critical Care Medicine, 27(4):337-349.

19. Guo Z, Li Q, Han Y, Liang Y, Xu Z, Ren T: Prevention of LPS-Induced Acute Lung Injury in Mice by Progranulin. Mediators of Inflammation, 2012:1-10.

20. Cao Y, Liu Y, Ping F, Yi L, Zeng Z, Li Y: miR-200b/c attenuates lipopolysaccharide-induced early pulmonary fibrosis by targeting ZEB1/2 via p38 MAPK and TGF- $\beta /$ smad3 signaling pathways. Laboratory Investigation.

21. Li C, Lei B, Huang S, Zheng M, Deng Y: H19 derived microRNA-675 regulates cell proliferation and migration through CDK6 in glioma. American Journal of Translational Research 2015, 7(10):17471764.

22. Long non-coding RNA H19 is responsible for the progression of lung adenocarcinoma by mediating methylation-dependent repression of $\mathrm{CDH1}$ promoter. Journal of Cellular and Molecular Medicine 
2019.

23. Cui J, Mo J, Luo M, Yu Q, Luo W: C-Myc-activated long non-coding RNA H19 downregulates miR-107 and promotes cell cycle progression of non-small cell lung cancer. International Journal of Clinical \& Experimental Pathology 2015, 8(10):12400-12409.

24. Sun H, Chen J, Qian W, Kang J, Wang J, Jiang L, Qiao L, Chen W, Zhang J: Integrated long non-coding RNA analyses identify novel regulators of epithelial-mesenchymal transition in the mouse model of pulmonary fibrosis. Journal of Cellular and Molecular Medicine, 20(7):1234-1246.

25. Liang H, Gu Y, Li T, Zhang Y, Huangfu L, Hu M, Zhao D, Chen Y, Liu S, Dong Y: Integrated analyses identify the involvement of microRNA-26a in epithelial-mesenchymal transition during idiopathic pulmonary fibrosis. Cell Death and Disease, 5(5):e1238.

26. Dunkel B: Acute Lung Injury and Acute Respiratory Distress Syndrome in Foals. Clinical Techniques in Equine Practice 2006, 5(2):127-133.

27. Becher RD, Colonna AL, Enniss TM, Weaver AA, Crane DK, Martin RS, Mowery NT, Miller PR, Stitzel JD, Hoth JJ: An innovative approach to predict the development of adult respiratory distress syndrome in patients with blunt trauma. Journal of Trauma and Acute Care Surgery, 73(5):1229-1235.

28. Xu T, Qiao J, Zhao L, Wang G, He G, Li K, Tian Y, Gao M, Wang J, Wang H: Acute Respiratory Distress Syndrome Induced by Avian Influenza A (H5N1) Virus in Mice. American Journal of Respiratory \& Critical Care Medicine, 174(9):1011-1017.

29. Xu X, Ning L, Yu-Xin Z, Jinjin C, Donglin W, Qisheng P, Hong-Bing W, Wan-Chun S: The Protective Effects of HJB-1, a Derivative of 17-Hydroxy-Jolkinolide B, on LPS-Induced Acute Distress Respiratory Syndrome Mice. Molecules, 21(1):77-

30. Felippe GADAC, Ribeiro SA, Patrícia B, Velho C-FM, Caire C-F-NH: Acute Respiratory Distress Syndrome: Role of Oleic Acid-Triggered Lung Injury and Inflammation. Mediators of Inflammation, 2015:1-9.

31. Hendrickson CM, Liu K, Calfee CS, Zhou H, Abbott J, Matthay MA: Higher Total Protein Concentration in Early Mini-BAL in ARDS Predicts Improved Clinical Outcomes.

32. Li W, Qi D, Lan C, Yan Z, Wang D-X: Vaspin protects against lipopolysaccharide-induced acute respiratory distress syndrome in mice by inhibiting inflammation and protecting vascular endothelium via PI3K/Akt signal pathway. Journal of Southern Medical University 2018, 38(3):283288.

33. Xin L, Wei C, Zhibo X, Qinghua H, Meiyan D: Therapeutic effect of ulinastatin on acute respiratory distress syndrome and its influence on serum levels of IL-6 and ICAM-1. Chinese Journal of Difficult and Complicated Cases 2017.

34. Zhai R, Gong MN, Zhou W, Thompson TB, Kraft P, Su L, Christiani DC: Genotypes and haplotypes of VEGF gene are associated with higher ARDS Mortality and lower VEGF plasma levels. 2007.

35. Chang L, Hong Z, Cheng PY, Zhou FC: [The influence of pre-B-cell colony enhancing factor on adhesive molecule in pulmonary cells in rats with acute lung injury/acute respiratory distress syndrome]. Zhonghua Wei Zhong Bing Ji Jiu Yi Xue 2013, 25(3):159-163. 
36. Wendel $M$, Giessmann $U$, Behrend $P$, Augstein $A$, Koch $T$ : Inflammatory-activated microvascular endothelial cells regulate interleukin-8 and monocyte chemoattractant protein-1 expression of $A 549$ cells in a paracrine fashion. Experimental Lung Research 2008, 34(2):85-100.

37. Howell DCJ, Chambers RC, Laurent GJ: Fibrosis in the Acute Respiratory Distress Syndrome. UPDATE IN INTENSIVE CARE AND EMERGENCY MEDICINE 2007.

38. Pozharskaya V, Torres-González E, Rojas M, Gal A, Amin M, Dollard S, Roman J, Stecenko AA, Mora AL: Twist: A Regulator of Epithelial-Mesenchymal Transition in Lung Fibrosis. PLOS ONE 2009, 4.

39. Li Y-Q, Xiang MR, Rong R, Lin H, Zhu QJ: Inhibitory effect of Glehniae Radix petroleum ether part on TGF- $\beta 1$-induced epithelial mesenchymal transition in A549 cells. China Journal of Chinese Materia Medica 2017, 42(9):1736-1741.

40. Xiaoguang Z, Jian S, Yingzhun C, Wei S, Huitong S, Yue L, Yining W, Nan Z, Hongli S, Haihai L: IncRNA PFAR Promotes Lung Fibroblast Activation and Fibrosis by Targeting miR-138 to Regulate the YAP1Twist Axis. Molecular Therapy:S1525001618302806-.

41. Yan W, Wu Q, Yao W, Li Y, Ni C: MiR-503 modulates epithelial-mesenchymal transition in silicainduced pulmonary fibrosis by targeting PI3K p85 and is sponged by InCRNA MALAT1. Scientific Reports 2017, 7(1):11313.

42. Huang C, Xiao X, Yang Y, Mishra A, Liu L: MicroRNA-101 attenuates pulmonary fibrosis by inhibiting fibroblast proliferation and activation. Journal of Biological Chemistry 2017, 292(40):jbc.M117.805747.

43. Ikari J, Nelson AJ, Obaid J, Giron-Martinez A, Rennard SI: Reduced microRNA-503 expression augments lung fibroblast VEGF production in chronic obstructive pulmonary disease. PLOS ONE 2017, 12(9):e0184039.

44. Y F, F G, J H, Z L: microRNA-1246 mediates lipopolysaccharide-induced pulmonary endothelial cell apoptosis and acute lung injury by targeting angiotensin-converting enzyme 2. 2017, 9(3):12871296.

45. Zeng Z, Gong H, Li Y, Jie K, Ding C, Shao Q, Liu F, Zhan Y, Nie C, Zhu W: Upregulation of miR-146a contributes to the suppression of inflammatory responses in LPS-induced acute lung injury. Experimental Lung Research, 39(7):275-282.

46. Lin-you L, Xi-ping H, Yuan-hui C, Department E: Effect of microRNA-132 transfection on lipopolysaccharide-induced inflammation in rat alveolar macrophages. Chinese Journal of Pathophysiology 2014, 30(12):2190-2194.

\section{Figures}



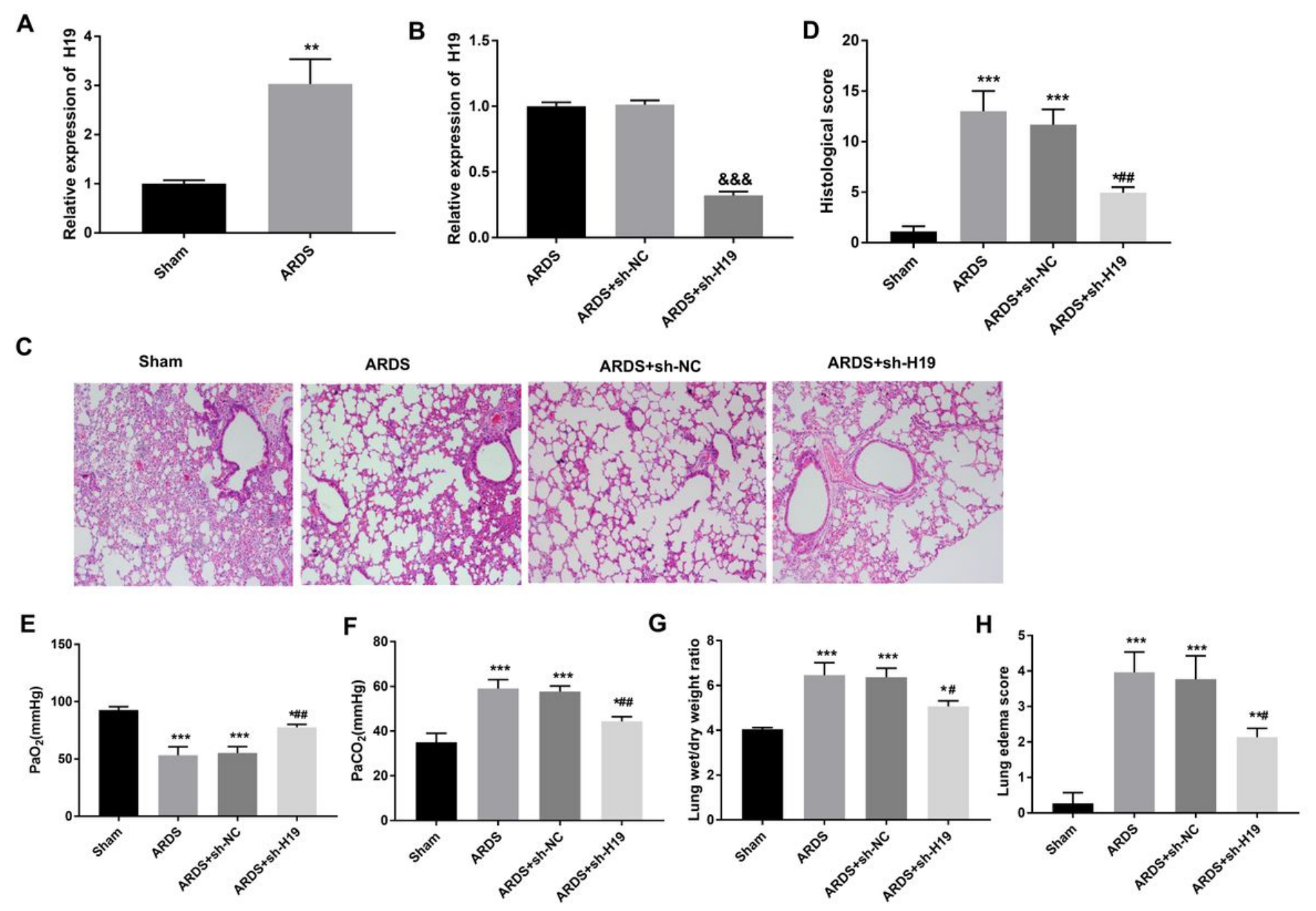

Figure 1

Silencing of $\mathrm{H} 19$ alleviates pulmonary injury of lipoproteins (LPS)-induced acute respiratory distress syndrome (ARDS). (A and B) The relative expression of $\mathrm{H} 19$ in lung tissues was detected by qRT-PCR. (C and $D$ ) Histopathological change and histology score were detected by hematoxylin-eosin (HE) staining. Original magnifications, $200 \times$. (E and F) Partial pressure of arterial oxygen (PaO2) and partial pressure of arterial carbon dioxide ( $\mathrm{PaCO} 2$ ) were measured using automatic blood gas analyzer. (G) Lung wet/dry weight ratio. $(H)$ Lung edema score. ${ }^{*} P<0.05$, ${ }^{\star *} P<0.01$, ${ }^{\star \star \star} P<0.001$ vs. Sham; \&\&\& $P<0.05$ vs. ARDS; \# $\mathrm{P}<0.05$, \#\# $\mathrm{P}<0.01$ vs. ARDS. 

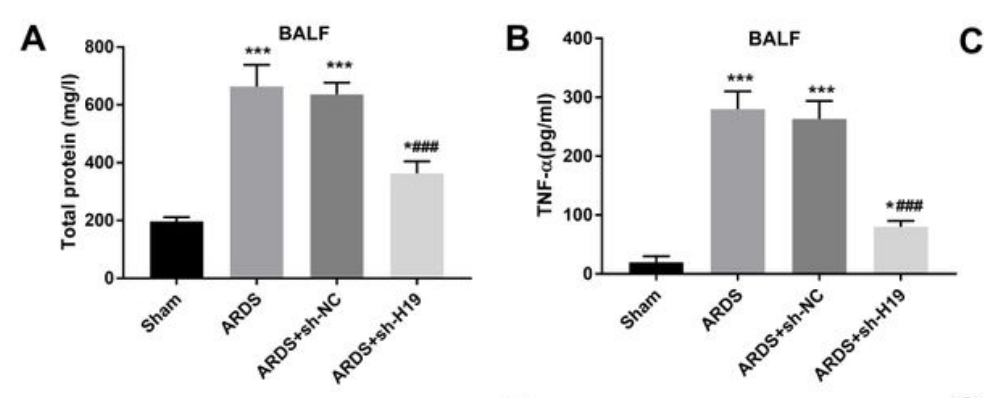

E
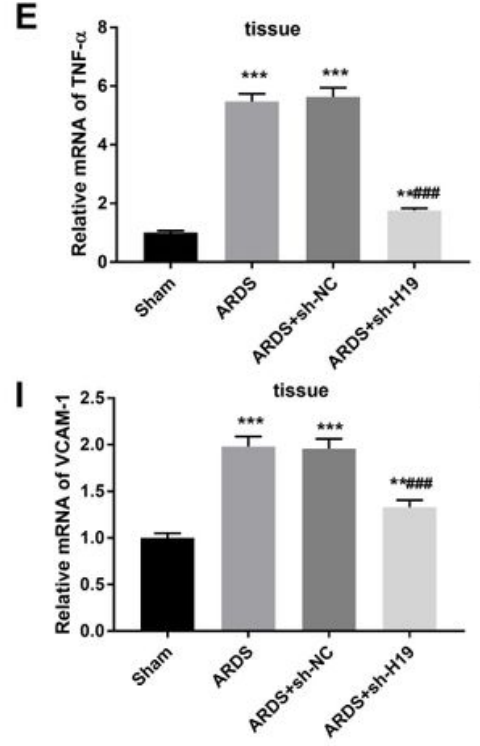

F

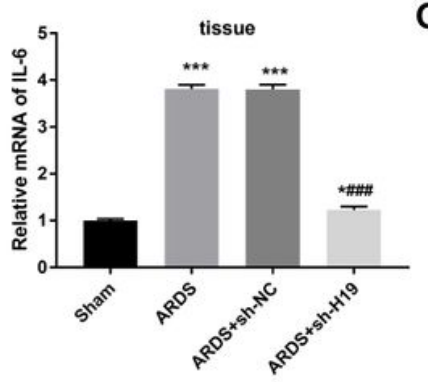

G

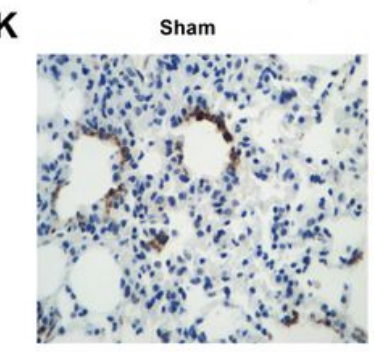

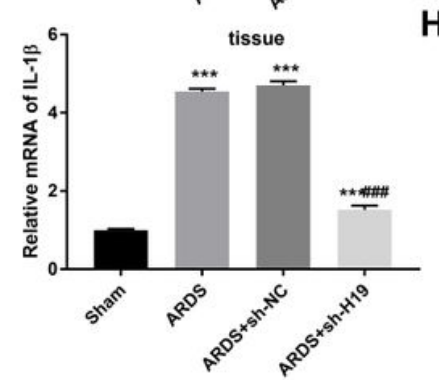

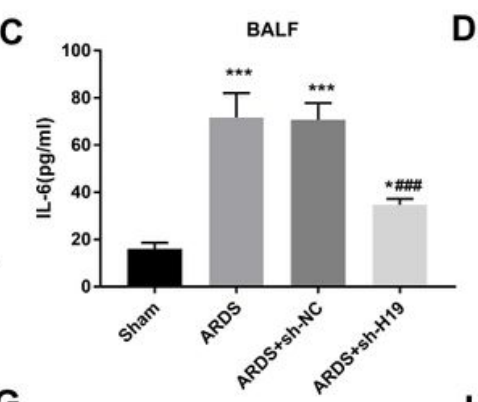

H

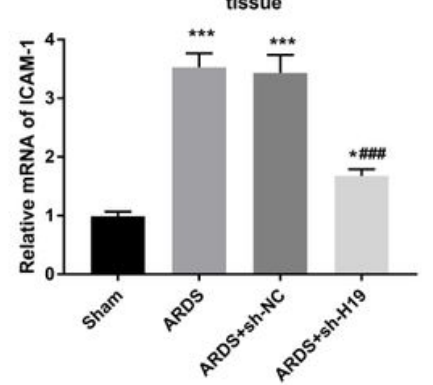

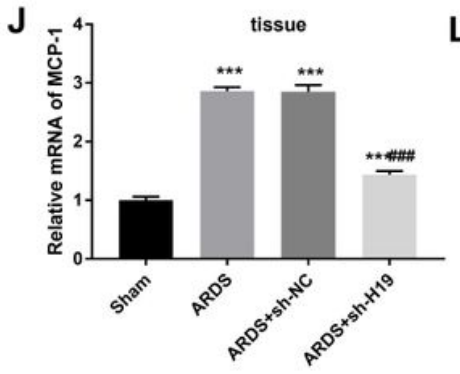
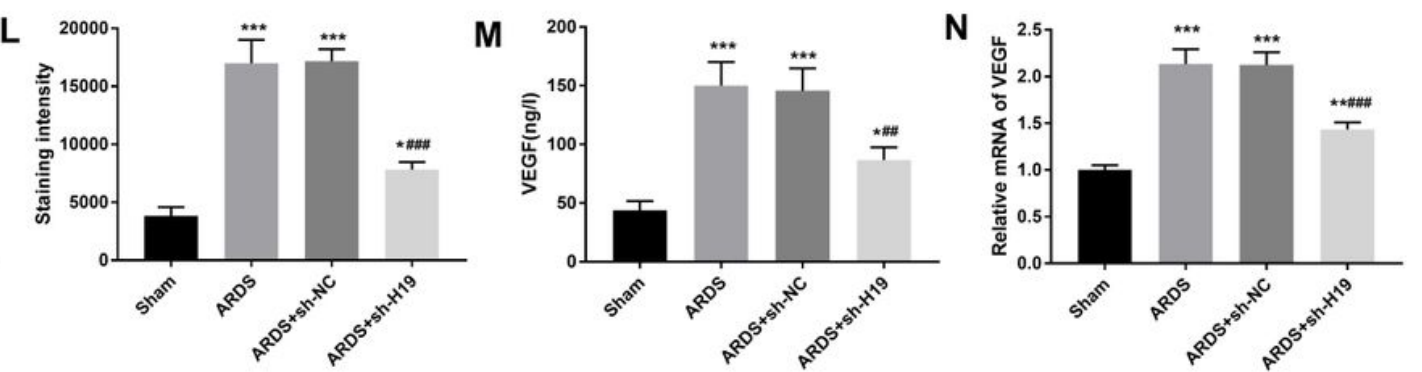

Figure 2

H19 inhibition attenuates pulmonary inflammation of lipoproteins (LPS)-induced acute respiratory distress syndrome (ARDS). (A-D) The levels of total protein (TP), tumor necrosis factor-a (TNF-a), interleukin (IL)- 6 and interleukin (IL)-1 $\beta$ in bronchoalveolar lavage fluid (BALF) were measured by ELISA. $(E-H)$ The relative mRNA expression of TNF- $a, I L-6, I L-1 \beta$, intercellular adhesion molecule-1 (ICAM-1), vascular cell adhesion molecule-1 (VCAM-1) and monocyte chemoattractant protein-1 (MCP-1) in lung tissues was measured by qRT-PCR. ( $K$ and L) The staining intensity of vascular endothelial growth factor (VEGF) was measured by immunohistochemical. Original magnifications, $400 \times$. (M) The concentration of VEGF in BALF was assessed by ELISA assay. (N) The relative mRNA expression of VEGF in lung tissues was measured by qRT-PCR. * $P<0.05$, ** $P<0.01$, *** $P<0.001$ vs. Sham; \#\# $P<0.01$, \#\#\# $P<$ 0.001 vs. ARDS. 

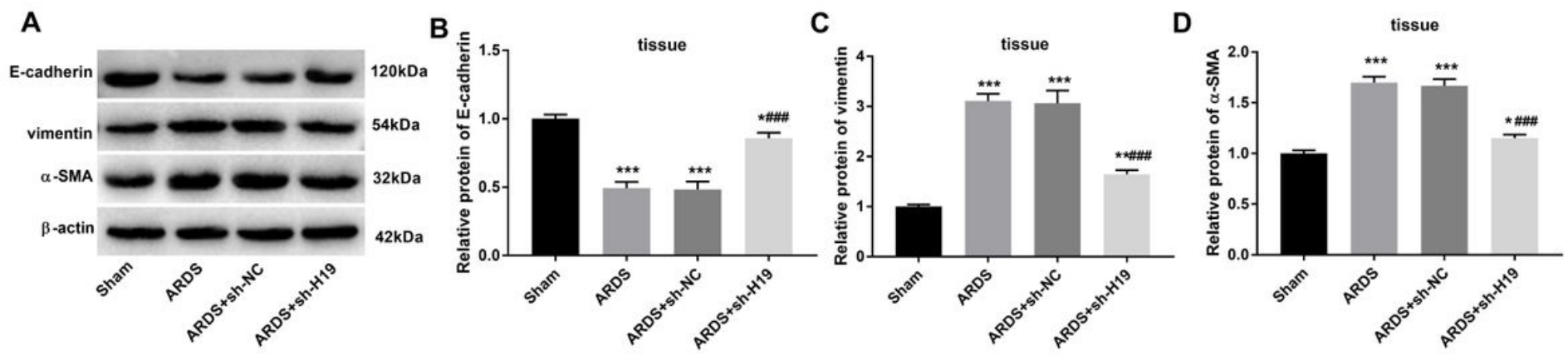

\section{E}
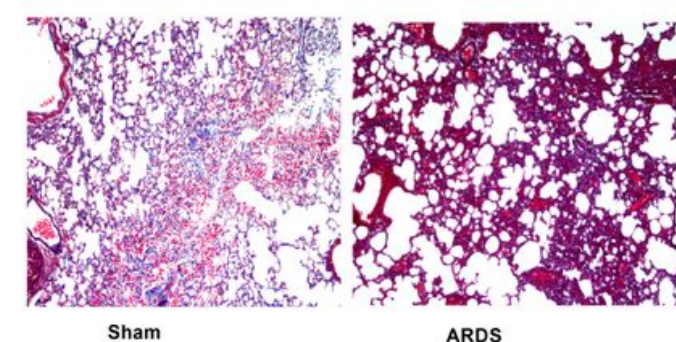

ARDS

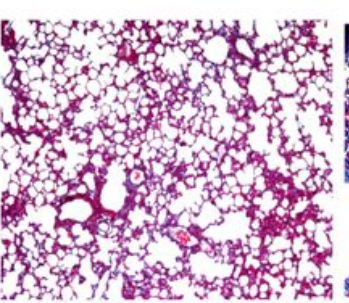

ARDS+sh-NC
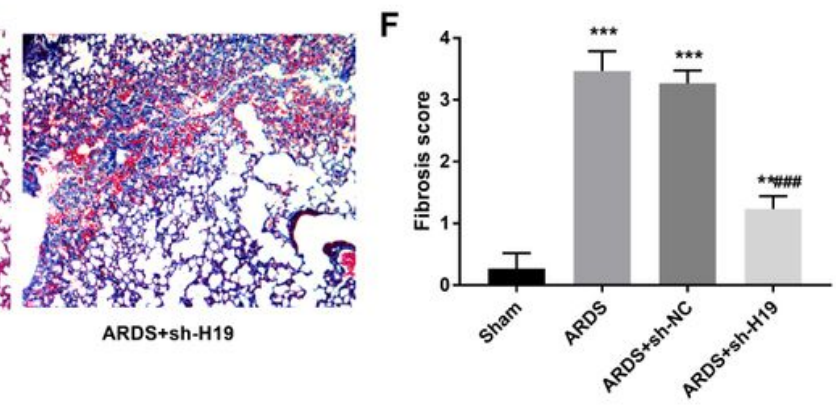

Figure 3

Knockdown of H19 ameliorates pulmonary fibrosis of lipoproteins (LPS)-induced acute respiratory distress syndrome (ARDS). (A) The protein bands of E-cadherin, vimentin and a-smooth muscle actin (aSMA). (B-D) The relative protein expression of E-cadherin, vimentin anda-SMA was measured by western blot. $(E)$ The ratio of fibrotic area was assessed by the masson-trichrome staining. Original magnifications, $200 \times$. (F) Fibrosis score. * $P<0.05$, ${ }^{\star *} P<0.01$, *** $P<0.001$ vs. Sham; \#\#\# $P<0.001$ vs. ARDS. 
A

H19 WT 5'-cccaUcucgCUCUGUGCCCCUC-3'

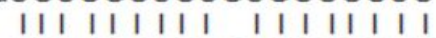

miR-423-5p 3'-uuucAGAGCGAGAGACGGGGAGu-5'

H19 MUT 5'-cccaAGAGCGAGAGACGGGGAG-3'
B

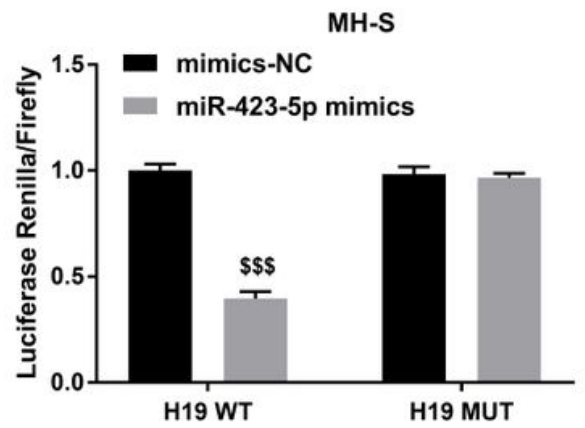

C

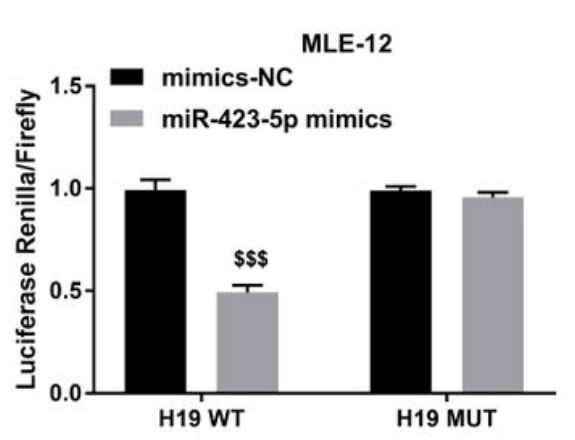

D

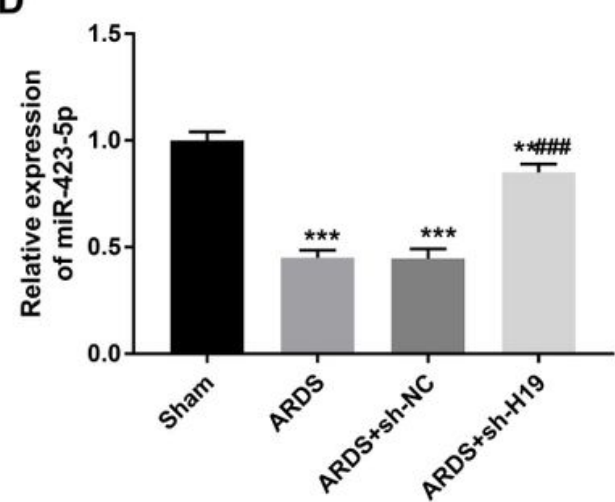

E

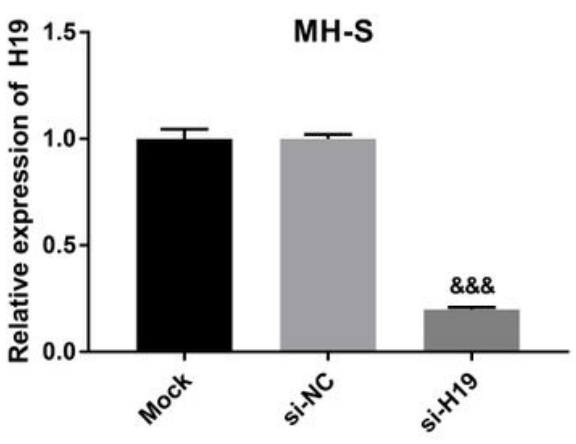

H
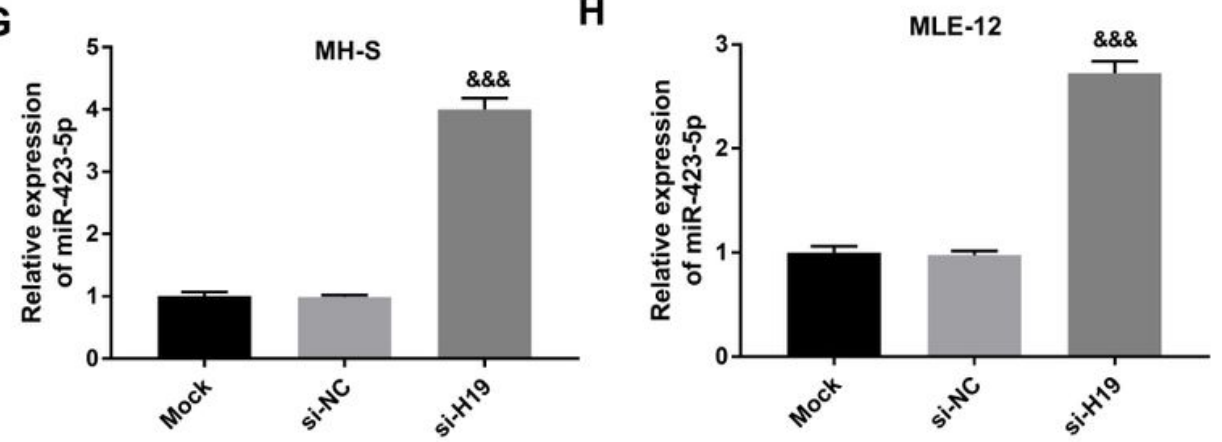

Figure 4

MiR-423-5p is a direct target of H19. (A) StarBase3.0 was used to predict the binding site between miR423-5p and H19. (B and C) Dual-luciferase reporter assay was performed to determine the luciferase activity of $\mathrm{MH}-\mathrm{S}$ and MLE-12 cells. (D) The relative expression of miR-423-5p in lung tissues was measured by qRT-PCR. (E-H) The relative expression of $\mathrm{H} 19$ and miR-423-5p in MH-S and MLE-12 cells was measured by qRT-PCR. \$\$ $P<0.001$ vs. mimics-NC; $* \star P<0.01$, *** $P<0.001$ vs. Sham; \#\#\# $P<$ 0.001 vs. ARDS; \&\&\& $P<0.05$ vs. Mock. 
A

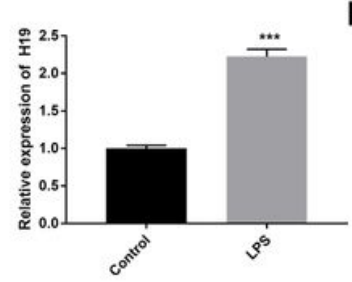

E

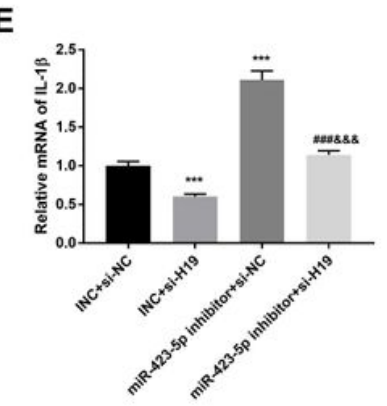

B

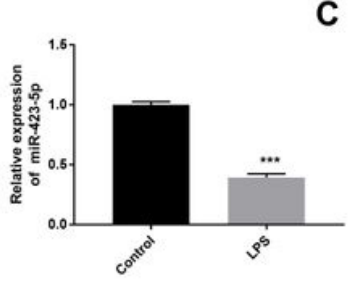

C

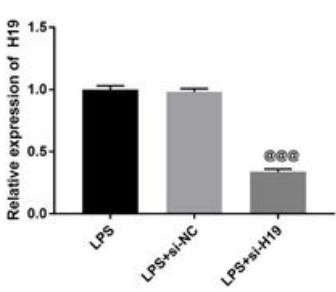

G

$\mathbf{F}$

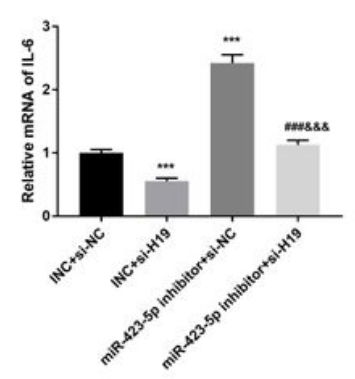

D

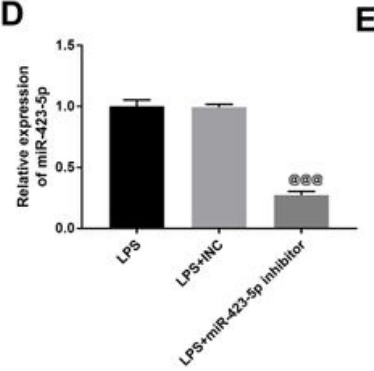

E

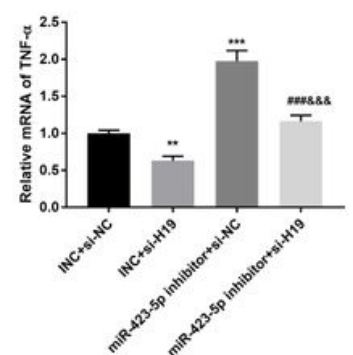

I

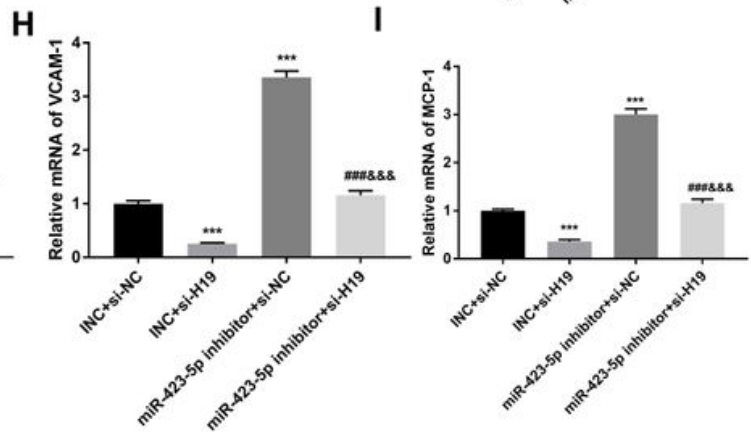

K

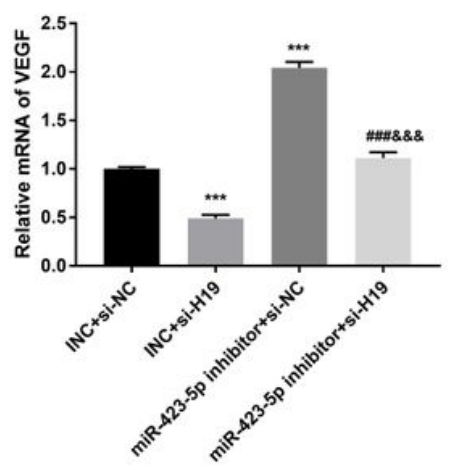

\section{Figure 5}

MiR-423-5p eliminates the effect of H19 on the lipoproteins (LPS)-induced MH-S cells. (A-D) The relative expression of $\mathrm{H} 19$ and miR-423-5p in MH-S cells was measured by qRT-PCR. (E-I) The relative mRNA expression of TNF-a, IL-6, IL-1 $\beta$, ICAM-1, VCAM-1 and MCP-1 in MH-S cells was measured by qRT-PCR. (J) The relative protein expression of E-cadherin, vimentin and a-SMA in MH-S cells was measured by western blot. (K) The relative mRNA expression of VEGF in MH-S cells was measured by $q R T-P C R$. ** $\mathrm{P}<$

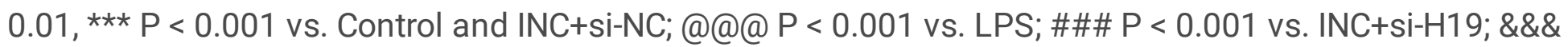
$\mathrm{P}<0.001$ vs. miR-423-5p inhibitor+si-NC.

\section{Supplementary Files}

This is a list of supplementary files associated with this preprint. Click to download.

- NC3RsARRIVEGuidelinesChecklistfillable1.pdf 